\title{
An Experimental Model of Neurodegenerative Disease Based on Porcine Hemagglutinating Encephalomyelitis Virus-Related Lysosomal Abnormalities
}

\author{
Yungang Lan ${ }^{1} \cdot \mathrm{Zi} \mathrm{Li}^{1} \cdot$ Zhenzhen Wang $^{1} \cdot$ Xinran Wang ${ }^{1} \cdot$ Gaili Wang $^{2} \cdot$ Jing Zhang $^{1} \cdot$ Shiyu Hu ${ }^{1} \cdot \mathrm{Kui} \mathrm{Zhao}^{1}$. \\ Baofeng $\mathrm{Xu}^{3} \cdot$ Feng $\mathrm{Gao}^{1} \cdot$ Wenqi He${ }^{1}$
}

Received: 19 May 2020 / Accepted: 27 August 2020 / Published online: 2 September 2020

(C) Springer Science+Business Media, LLC, part of Springer Nature 2020

\begin{abstract}
Lysosomes are involved in pathogenesis of a variety of neurodegenerative diseases and play a large role in neurodegenerative disorders caused by virus infection. However, whether virus-infected cells or animals can be used as experimental models of neurodegeneration in humans based on virus-related lysosomal dysfunction remain unclear. Porcine hemagglutinating encephalomyelitis virus displays neurotropism in mice, and neural cells are its targets for viral progression. PHEV infection was confirmed to be a risk factor for neurodegenerative diseases in the present. The findings demonstrated for the first time that PHEV infection can lead to lysosome disorders and showed that the specific mechanism of lysosome dysfunction is related to PGRN expression deficiency and indicated similar pathogenesis compared with human neurodegenerative diseases upon PHEV infection. Trehalose can also increase progranulin expression and rescue abnormalities in lysosomal structure in PHEV-infected cells. In conclusion, these results suggest that PHEV probably serve as a disease model for studying the pathogenic mechanisms and prevention of other degenerative diseases.
\end{abstract}

Keywords Neurodegenerative diseases · Porcine hemagglutinating encephalomyelitis virus - Lysosomal abnormalities · Progranulin · Trehalose

$\begin{array}{ll}\text { Abbreviations } \\ \text { PHE } & \text { Porcine hemagglutinating encephalomyelitis } \\ \text { PHEV } & \text { Porcine hemagglutinating encephalomyelitis virus } \\ \text { PGRN } & \text { Progranulin } \\ G R N & \text { Granulin } \\ \text { N2a } & \text { Neuro-2a } \\ \text { CNS } & \text { Central nervous system } \\ \text { RABV } & \text { Rabies virus }\end{array}$

Yungang Lan and $\mathrm{Zi} \mathrm{Li} \mathrm{contributed} \mathrm{equally} \mathrm{to} \mathrm{this} \mathrm{work.}$

Electronic supplementary material The online version of this article (https://doi.org/10.1007/s12035-020-02105-y) contains supplementary material, which is available to authorized users.

\section{Wenqi He}

hewq@jlu.edu.cn

1 Key Laboratory of Zoonosis Research, Ministry of Education, College of Veterinary Medicine, Jilin University, Changchun, China

2 Jilin Academy of Animal Husbandry and Veterinary Medicine, Changchun, Jilin, China

3 Department of Neurosurgery, The First Hospital of Jilin University, Changchun 130021, China

$\begin{array}{ll}\text { ZIKV } & \text { Zika virus } \\ \text { A } \beta & \text { Amyloid- } \beta \\ \alpha-S Y N & \text { A-synuclein } \\ \text { NCL } & \text { Neuronal ceroid lipofuscinosis } \\ \text { FTLD } & \text { Frontotemporal lobar degeneration } \\ \text { TDP-43 } & \text { The transactive response DNA binding protein 43 } \\ \text { GAPDH } & \text { Glyceraldehyde-3-phosphate dehydrogenase } \\ \text { TCID } 50 & 50 \% \text { median tissue culture infectious dose } \\ \text { QPCR } & \text { Quantitative reverse transcription-PCR analysis } \\ \text { PBS } & \text { Phosphate-buffered saline } \\ \text { CTSD } & \text { Cathepsin D } \\ \text { AD } & \text { Alzheimer's disease } \\ \text { PD } & \text { Parkinson's disease } \\ \text { FTD } & \text { Frontotemporal dementia } \\ \text { ALS } & \text { Amyotrophic lateral sclerosis } \\ \text { H } & \text { Hour }\end{array}$

\section{Background}

Neurodegeneration is a characteristic of many debilitating, incurable diseases that are rapidly increasing in prevalence 
in humans. There is an urgent need to develop new and more effective therapeutic strategies to combat these devastating diseases. Models ranging from cell-based systems to unicellular organisms and complex animals have proven to be a useful tool shedding light on the mechanisms underlying neurodegenerative diseases, and these advances have now begun to reveal promising therapeutic avenues [1]. A variety of neurotropic virus infections of the central nervous system, especially those characterized by a chronic progressive course, may produce multiple incidences of damage in infected and neighboring neurons; this damage is a possible risk factor for neurodegenerative diseases such as those caused by rabies virus (RABV) [2] and Zika virus (ZIKV) [3]. A growing body of epidemiologic and experimental data indicates that viral infections cause chronic damage resulting in alterations of neuronal function and viability by directly triggering neurotoxic pathways or the activation of immune responses. Indeed, viral agents have been reported to induce molecular hallmarks of neurodegeneration such as the production and deposition of misfolded protein aggregates, including amyloid- $\beta(A \beta)$ [4] and a-synuclein $(\alpha-\mathrm{SYN})$ [5]. The questions of whether the pathogenesis of these infections shows similarity to that of human neurodegenerative diseases and whether virusinfected cells or animals can be used as experimental models of neurodegeneration in humans remain under debate.

Porcine hemagglutinating encephalomyelitis (PHE) is an infectious disease of naïve pigs, in which motor disorders are often seen during field outbreaks, but the clinical disease is variable and dependent on age. This disease is caused by porcine hemagglutinating encephalomyelitis virus (PHEV), a single-stranded, nonsegmented, positive-stranded RNA coronavirus belonging to the Betacoronavirus genus within the Coronaviridae family, subfamily Cornavirinae, order Nidovirales [6]. PHEV also displays neurotropism in mice and Wistar rats and produces acute encephalomyelitis [7, 8]. The results of previous in vivo or in vitro studies using mice or neuro-2a (N2a) cells have suggested that PHEV invades the central nervous system (CNS) via the peripheral nervous system (i.e., neural spread) and infects nerve cells. Typical neurological symptoms of emaciated PHEV-infected mice include generalized muscle tremors, hyperesthesia, movements of the front and hind feet similar to piano playing, and a tendency to sit in a dog-like position [9]. The neuronal bodies show very little pathological change, but obvious degeneration of neuronal processes occurs after infection with pathogenic PHEV, including stunted axon elongation, unstable dendritic spine formation, and disconnection of neurites $[10,11]$. These observations indicate that PHEV infection is a risk factor for neurodegenerative diseases. In the present study, we first found that the pathogenesis of PHEV infection shows similarity to that of human neurodegenerative diseases, and PHEV may serve as a disease model for studying the pathogenic mechanisms and prevention of other degenerative diseases.

\section{Materials and Methods}

\section{PHEV, Cells Lines, Antibodies, and Chemical Reagents}

The PHEV stain used in this study was PHEV CC14 (GenBank accession number MF083115.1), which was previously kept by our research group. The mouse neuroblastoma cell line neuro-2a (N2a from ATCC) were infected with PHEV $\left(10^{4.50} 50 \%\right.$ median tissue culture infectious dose $\left[\mathrm{TCID}_{50}\right] / 0.1 \mathrm{~mL}$ ) in cell cultures with $2 \%$ FBS for 1 hour (h). Following a 1-h absorption period, infected cells were incubated in the complete DMEM at $37{ }^{\circ} \mathrm{C}$ for the relevant times in the conformity with experimental requirements. EGFP/EGFP-PGRN-overexpressing mouse neuroblastoma cell (N2a cell) lines were generated by our research group. The mouse antiPHEV-S antibody was our laboratoryprepared monoclonal antibody [12]. The following primary antibodies were used in this study: rat antimouse LAMP1 antibody (553792) from BD Biosciences, sheep antiGRN/ progranulin antibodies from R\&D systems (AF2557), rabbit antiTDP-43 antibodies (0782-2-AP), rabbit antiCTSD antibodies (21327-1-AP), and mouse antiGFP antibodies (66002-1-Ig) from Proteintech. The following secondary antibodies were used: donkey antimouse Alexa Fluor 594 (A21203), donkey antirabbit Alexa Fluor 488 (A21206), donkey antimouse Alexa Fluor 647 (A31571), donkey antisheep Alexa Fluor 647 (A21448), and Hoechst stain (H1339) from Thermo Fisher Scientific. Trehalose was obtained from Sigma and was dissolved in ultrapure Milli-Q water (EMD Millipore) [13].

\section{Animal Protocols}

Three weeks old BALB/c mice (male) were obtained from the Laboratory Animal Center of Jilin University. The PHEVinfected model was established in mice $[9,11]$. Briefly, the mice were anaesthetized with isoflurane and were intranasally (i.n.) inoculated with $0.1 \mathrm{ml}$ of virus $\left(10^{4.50} \mathrm{TCID}_{50}\right)$ diluted in phosphate-buffered saline (PBS, $1 \mathrm{M}, \mathrm{PH} 7.4$ ). Mice mock infected i.n. with $0.1 \mathrm{ml}$ PBS were used as controls. Mice were euthanized at 4-day postinfection (p.i) by CO 2 inhalation according to animal handling laws.

\section{Brain Tissue Sectioning and Staining}

For immunofluorescence staining, fresh brain tissues from PHEV-infected or mock-infected mice frozen in liquid nitrogen and embedded in OCT compound were cut into cryostat sections $(10 \mu \mathrm{m})$ and mounted on Superfrost Plus slides. Before staining, the slides were warmed at room temperature for $60 \mathrm{~min}$ and were then immersed in blocking buffer (5\% nonfat milk and $0.05 \%$ Triton X-100 in PBS) for $1 \mathrm{~h}$ at room temperature. Slides were then treated with primary antibodies 
diluted in blocking buffer overnight at $4{ }^{\circ} \mathrm{C}$, and subsequently incubated with secondary antibodies conjugated with either Alexa 488, Alexa 594, or Alexa 647 for $2 \mathrm{~h}$ at room temperature. After counterstaining the samples, the coverslips were mounted and viewed under the Olympus FV1000 confocal microscope (Olympus, Japan).

\section{Immunocytochemistry}

PHEV-infected or mock-infected cells grown on glass coverslips were fixed in $4 \%$ paraformaldehyde for $15 \mathrm{~min}$, washed 3 times with PBS, and permeabilized and blocked in blocking buffer for $15 \mathrm{~min}$. Primary antibodies diluted in blocking buffer were applied to the cells overnight at $4{ }^{\circ} \mathrm{C}$. Coverslips were washed 3 times with PBS. Secondary antibodies and Hoechst stain diluted in blocking buffer were applied to the cells for $2 \mathrm{~h}$ at room temperature. Coverslips were viewed under the Olympus FV1000 confocal microscope (Olympus, Japan).

\section{Quantitative Reverse Transcription-PCR Analysis}

For qPCR, RNA was purified from cells using TRIzol reagent (Thermo Fisher Scientific). Two micrograms of total RNA was reverse transcribed using a poly $(\mathrm{T})$ primer and SuperScript III Reverse Transcriptase (Thermo Fisher Scientific). The primer sequences used in these assays are shown in Fig. 1c. qPCR was performed in a LightCycler 480 (Roche Applied Science), and the thermocycler amplification conditions were $94^{\circ} \mathrm{C}$ for $5 \mathrm{~min}$, and 40 cycles of $95^{\circ} \mathrm{C}$ for $15 \mathrm{~s}, 59^{\circ} \mathrm{C}$ for $30 \mathrm{~s}$, and $72{ }^{\circ} \mathrm{C}$ for $10 \mathrm{~s}$. Transcript levels were calculated using the efficiency-adjusted $\Delta \Delta$-CT method. All transcripts were normalized to GAPDH [14].

\section{Immunoprecipitation}

Cells were washed with PBS at $48 \mathrm{~h}$ postPHEV infection, and the cell lysates were collected in IP buffer $(50 \mathrm{mM}$ Tris $\mathrm{pH}$ $8.0,150 \mathrm{mM} \mathrm{NaCl}, 1 \%$ Triton, $0.1 \%$ deoxycholate with protease inhibitors) as described previously [14]. The lysates were immunoprecipitated using GFP-trap beads for $4 \mathrm{~h}$ as described previously [15].

\section{Protein Analysis}

Cells and tissues were lysed in RIPA buffer ( $50 \mathrm{mM}$ Tris, $\mathrm{pH}$ 8.0, $150 \mathrm{mM} \mathrm{NaCl}, 1 \%$ Triton X-100 (Sigma-Aldrich, 78787), $0.1 \%$ SDS, $0.1 \%$ deoxycholic acid (Sigma-Aldrich, 83-44-3) with protease and phosphatase inhibitors (SigmaAldrich, P5726). After being separated by SDS-PAGE, samples were run on $12 \%$ polyacrylamide gels and transferred to PVDF membranes (Millipore, IPFL00010) and then immunoblotted with the indicated antibodies. Western blot signals were then analyzed using the Image $\mathrm{J}$ software.

\section{Image Analysis}

For the quantification of enlarged lysosomes, the lysosomes were visualized by antiLAMP1 staining, and N2a were captured using Z stack. Cells with enlarged lysosomes (diameter> $1 \mu \mathrm{m})$ were counted. It should be noted that during fixation, lysosomal size, and area might have been changed, but we always have a control group and experimental group analyzed at the same time [14]. For PGRN and LAMP1 colocalization, background was subtracted, and the colocalization between the PGRN and LAMP1 fluorescence was determined by Manders' Coefficients using Plugins 'JACOP' of ImageJ software [15].

\section{Statistical Analysis}

The data were presented as mean \pm SEM. Two-group analysis was performed using the Student's $t$ test. *, $P$ values $<0.05$ were considered statistically significant. $* *, P$ values $<0.01$ were considered statistically very significant.

\section{Result and Discussion}

Lysosomes are membrane-bound organelles that play roles in the degradation and recycling of cellular waste. Lysosomal dysfunction tends to affect the central nervous system (CNS) to a greater extent than that of other organelles because the maintenance of adequate lysosomal function is especially important for the health of postmitotic neurons in the CNS that are destined to survive the entire lifetime of the organism. Lysosomal dysfunction and defects in fusion with vesicles containing cargo are commonly observed abnormalities in proteinopathic disorders that lead to protein aggregates, which are a common pathological feature of neurodegenerative disease $[16,17]$. In addition, the lysosomes are involved in the exclusion of infectious agents from the penetration of host tissue and concomitant immune regulation and must therefore be able to respond quickly with increased or decreased function to various metabolic conditions, with the aim of to protecting cells from death or damage upon virus infection [18]. Therefore, lysosomes play a large role in neurodegenerative disorders caused by virus infection. To test the roles of lysosomes in the PHEV infection process, N2a cells were infected with PHEV. The cells were fixed, and the lysosomes and PHEV were visualized by antiLAMP1 and antiPHEV staining. We found that PHEV localized to the lysosomes and resulted in enlarged lysosomes (Fig 1a). Indeed, PHEV infection resulted in increased LAMP1 expression levels in vitro [19] and increased lysosome enlargement in vivo (Additional file 1). These results showed that PHEV infection leads directly to lysosomal dysfunction and indicated that there was a similarly pathological feature between PHE and 

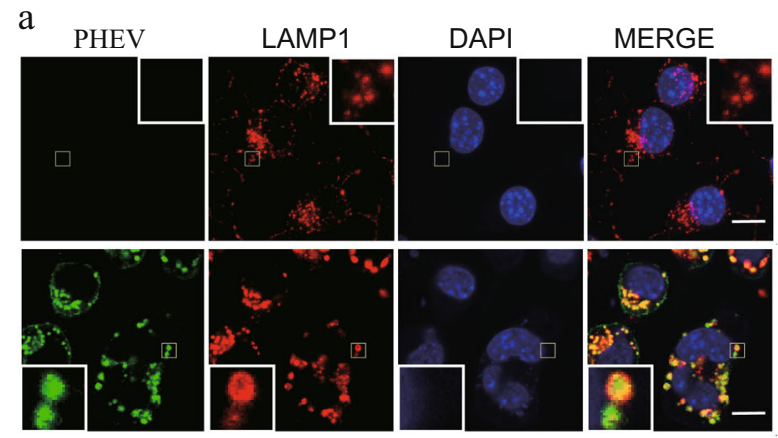

b
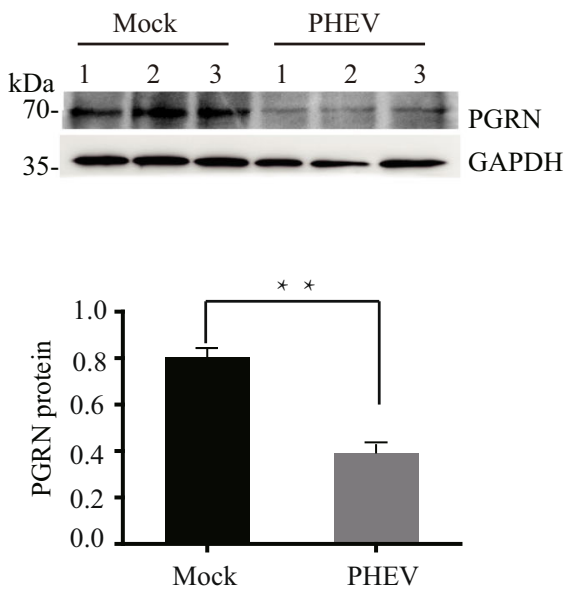

d
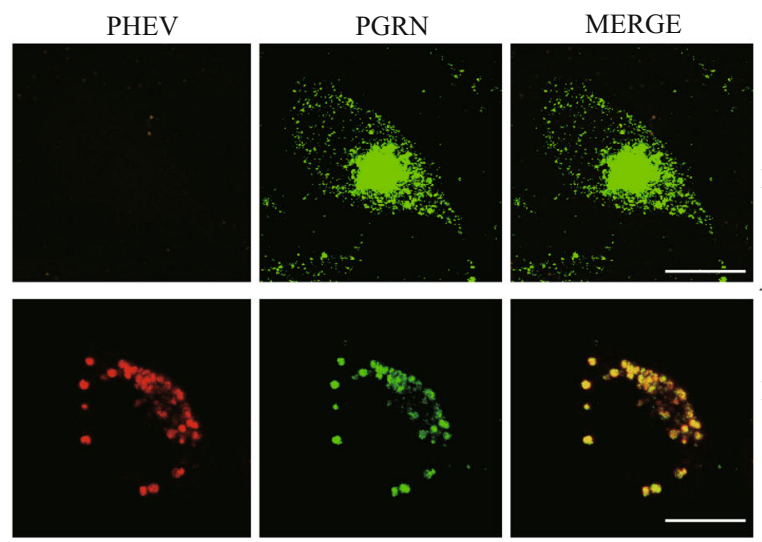

Fig. 1 PHEV infection leads to lysosomal abnormalities. a PHEV localizes to lysosomes, and PHEV infection results in greater lysosome enlargement compared with mock infection. The percentage of $\mathrm{N} 2 \mathrm{a}$ cells containing enlarged lysosomes $(>1 \mu \mathrm{m})$ was quantified in the experiment. Scale bar $=10 \mu \mathrm{m}$. All the results are presented as the means \pm the $\mathrm{SD}$ of the data from three independent experiments $(* *, P$ $<0.01)$. b PHEV infection decreased PGRN protein expression. PGRN protein levels in PHEV-infected or mock-infected N2a cells were quantified and normalized to GAPDH. $n=3$; one-way ANOVA; **, $P<0.01$;

several neurodegenerative diseases. Further studies will be needed to elucidate the underlying molecular mechanisms of lysosomal dysfunction by PHEV infection in vitro or in vivo.

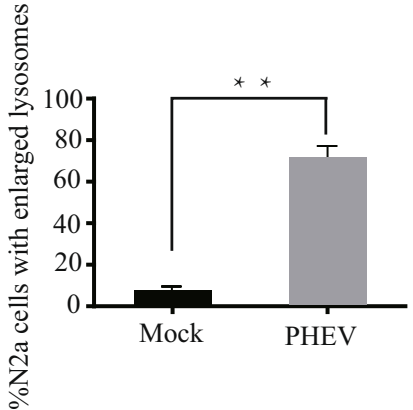

$\mathrm{c}$

\begin{tabular}{cc}
\hline Name & Primer \\
\hline & Forward 5'-AGTTCGAATGTCCTGACTCCGCCA-3' \\
PGRN & Reverse 5'-AAGCCACTGCCCTGTTGGTCCTTT-3' \\
& Forward 5'-CTCAACTACATGGTCTACATGTTC-3' \\
GAPDH & Reverse 5'-ATTTGATGTTAGTGGGGTCTCGCTC-3' \\
\hline
\end{tabular}
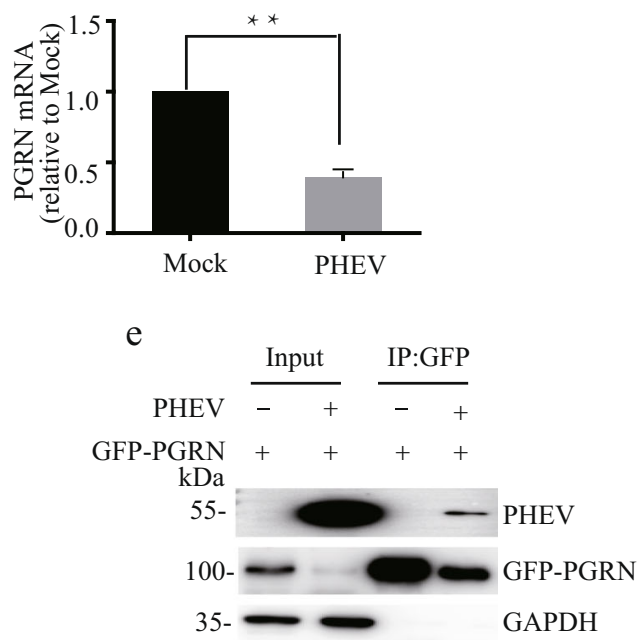

PHEV

Student's $t$ test. c PHEV infection decreased PGRN mRNA expression. PGRN mRNA levels in PHEV-infected or mock-infected N2a cells were quantified and normalized to GAPDH. $n=3$; one-way ANOVA; $* *, P<$ 0.01 ; Student's $t$ test. d PHEV bound to PGRN. PHEV-infected or mockinfected N2a cells were stained with antiPHEV (red) and antiPGRN (green). Scale bar $=10 \mu \mathrm{m}$. e AntiGFP immunoprecipitates from PHEV-infected or mock-infected EGFP-PGRN-transfected N2a cells were harvested, and the physical interaction between PGRN and PHEV was demonstrated

The progranulin (PGRN) protein, encoded by the granulin $(G R N)$ gene, has been recently implicated in several neurodegenerative diseases [20,21]. PGRN localizes to the lysosome and is important for proper lysosomal structure and function. 

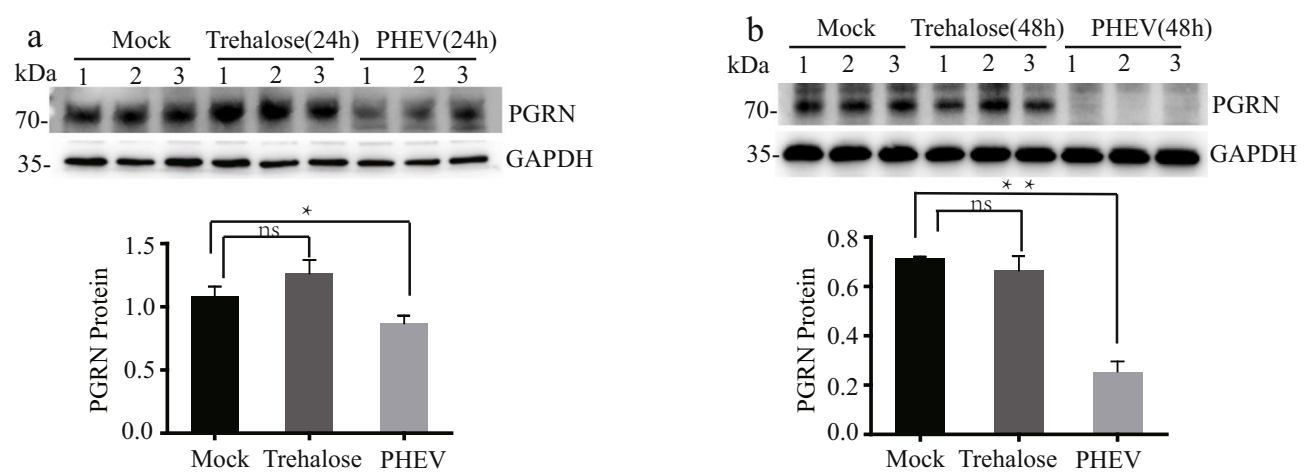

c
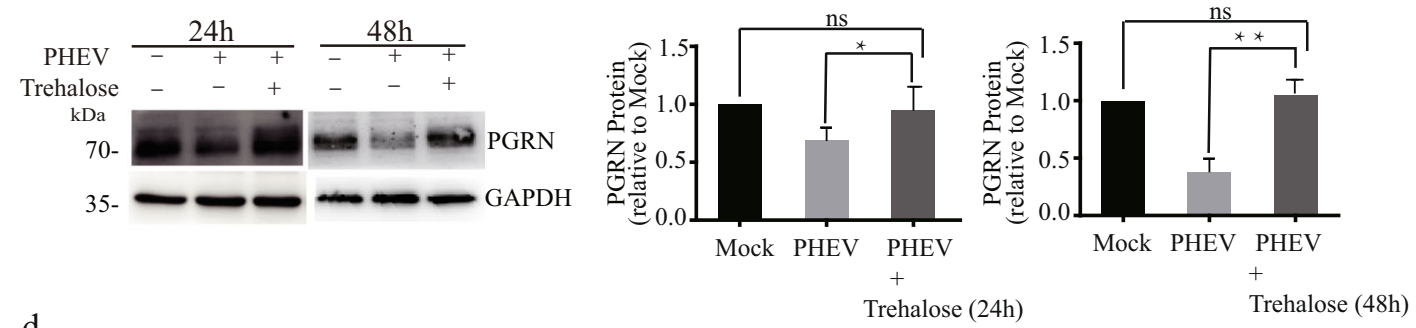

d
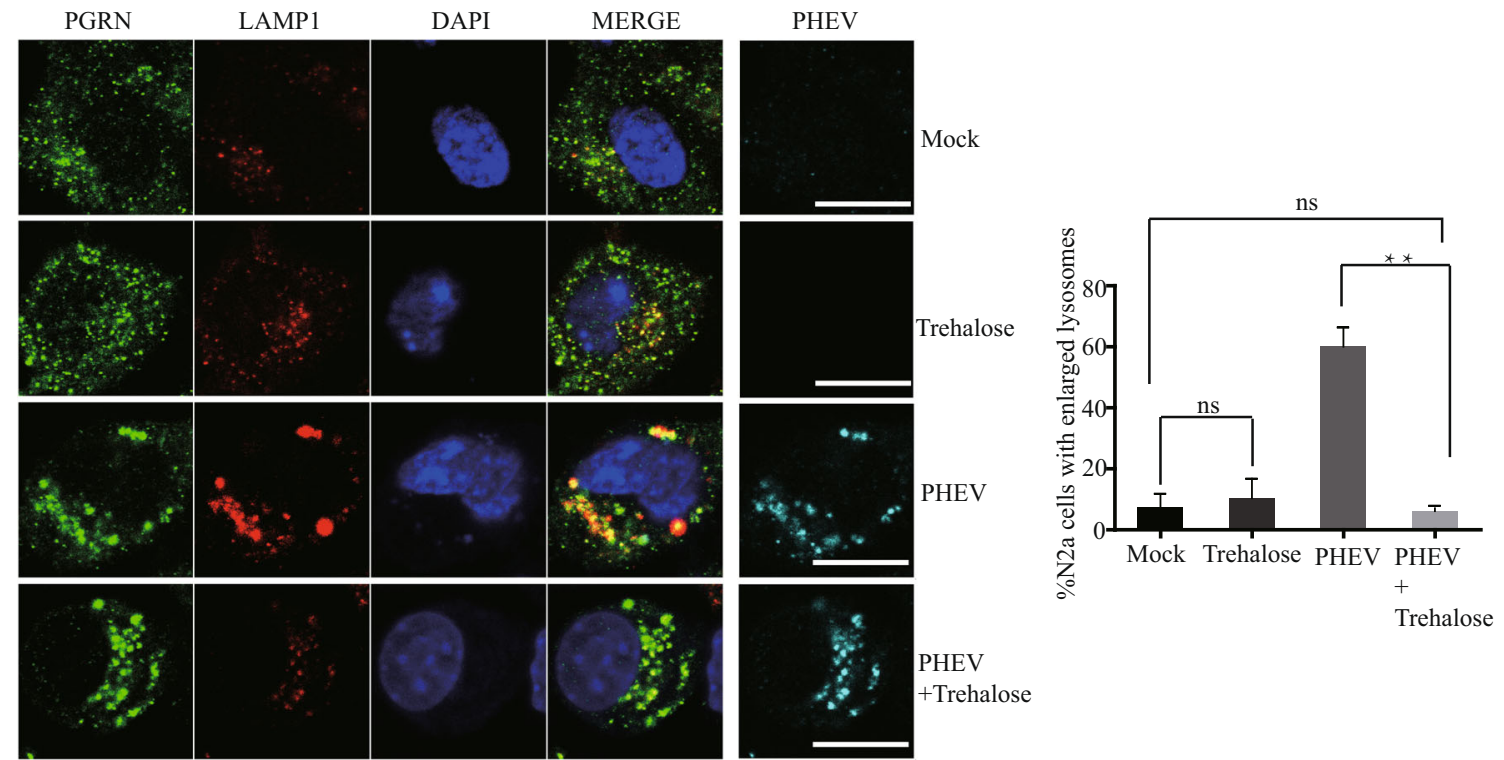

Fig. 2 Trehalose increases PGRN expression and rescue abnormalities in lysosomal structure in PHEV-infected cells. a-c Trehalose did not influence PGRN expression in normal cells but increased PGRN expression in PHEV-infected cells. PGRN protein levels in PHEV- or mock-infected cells that were treated or not with $100 \mathrm{mM}$ trehalose for $24 \mathrm{~h}$ or $48 \mathrm{~h}$ were quantified and normalized to GAPDH. $n=3$, one-way ANOVA; *, $P<$

Strong evidence has shown that lysosomal dysfunction occurs in cases of PGRN deficiency [22, 23]; haploinsufficiency results in frontotemporal lobar degeneration (FTLD), and complete loss results in neuronal ceroid lipofuscinosis (NCL) [24, 25]. To examine the change in PGRN expression in PHEVinfected N2a cells, cell lysates of PHEV-infected normal or EGFP-PGRN-overexpressing N2a cells were analyzed by

0.05 ; **, $P<0.01$; Student's $t$ test. d There were fewer enlarged lysosomes in PHEV-infected cells treated with $100 \mathrm{mM}$ trehalose compared to untreated cells treated with trehalose for $48 \mathrm{~h}$. The percentage of N2a cells containing enlarged lysosomes $(>1 \mu \mathrm{m})$ was quantified in the experiment. All the results are presented as the means \pm the SD of the data from three independent experiments $(* *, P<0.01)$. Scale bar $=10 \mu \mathrm{m}$

western blotting with antiPGRN or antiGFP antibodies. The results showed that both endogenous and exogenous PGRN expression was obviously decreased by PHEV infection compared to mock infection (Fig. 1b, Additional file 2), and PHEV infection resulted in PGRN expression deficiency in vivo (Additional file 3 ). Indeed, qPCR analysis also showed decreased mRNA levels of PGRN in PHEV-infected N2a 
cells (Fig. 1c). Furthermore, these cells were fixed, and the lysosomes, PGRN, and PHEV were visualized by antiLAMP1, antiPGRN, and antiPHEV staining, respectively. The results showed that PHEV bound to PGRN and that PGRN was passively transported to lysosomes because of PHEV infection (Fig. 1d, Additional file 4). To test the physical interaction between PGRN and PHEV, PHEV- or mockinfected EGFP-PGRN-transfected N2a cells were used. Cell lysates were immunoprecipitated with antiGFP beads. A PHEV signal is detected in PHEV-infected immunoprecipitates but not under mock infection (Fig. 1e), suggesting a physical interaction between PGRN and PHEV. PGRN can be intracellularly processed into $10 \mathrm{kDa}$ peptides in multiple cell types, and this processing is dependent on lysosomal activities [24]. However, we found that PGRN was not passively cleaved in the abnormal lysosomes of PHEV-infected cells (data not shown). These results showed that PHEV infection could lead to lysosome dysfunction by influencing PGRN expression and lysosome trafficking and indicated similar pathogenesis compared with human neurodegenerative diseases such as NCL and FTLD upon PHEV infection. However, the role of PGRN in lysosomal dysfunction and the cause of PGRN deficiency induced by PHEV infection still need further study.

Furthermore, increasing evidence indicates that upregulated lysosomal function in neurons represents a promising therapeutic approach for neurodegenerative disorders by targeting lysosomal proteins and processes, particularly with small molecules and peptide drugs [26, 27]. Recent advances in the understanding of PGRN biology emphasize its roles in lysosomal function and indicate that increasing PGRN levels is a potential therapeutic approach for multiple neurodegenerative diseases [26]. A recent study demonstrated that trehalose could upregulate progranulin expression in human and mouse models of PGRN deficiency, as a novel therapeutic strategy for treating frontotemporal dementia (FTD) [13]. Therefore, we tried to verify the treatment effect of trehalose, which did not influence PGRN expression in normal cells, by using models from N2a cell-based systems after PHEV infection (Fig. 2a, b). Our results showed that trehalose function was successfully verified by increasing PGRN expression in PHEV-infected N2a cells (Fig. 2c). Indeed, trehalose simultaneously caused a decrease in enlarged lysosomes (Fig. 2d) and inhibited virus replication under PHEV infection (data not shown). However, whether trehalose has a similar therapeutic effect in PHEV-infected mice is still unclear.

It is known that the disease model is a theory concerned with the cause and course of a pathological condition or process and, at last, implications for clinical practice [1, 28]. Indeed, models based on pathogenic gene expression disorders have provided both insight into molecular mechanisms and the temporality of changes of the human disease and helped to identify candidate, potentially disease modifying, therapies. We first demonstrated that PHEV infection can lead to lysosome disorders and showed that the specific mechanism of lysosome dysfunction is related to PGRN expression deficiency (Fig. 1). Trehalose can also increase PGRN expression and rescue abnormalities in lysosomal structure in PHEV-infected cells (Fig. 2). Furthermore, cathepsin D (CTSD) is the main lysosomal enzyme involved in the degradation of various pathogenic proteins. We found a possible new effect of trehalose on increasing the procathepsin D (ProCTSD) and mature cathepsin D (Mat-CTSD) expression by using models from N2a cell-based systems after PHEV
Fig. 3 PHEV infection increases TDP-43 protein expression. a TDP-43 levels were increased in the brain of PHEV-infected mice. TDP-43 protein levels in the brain of PHEV-infected or mockinfected mice were quantified and normalized to GAPDH. $n=3$; one-way ANOVA; *, $P<0.05$; Student's $t$ test. b Immunoblot analysis of PHEV and TDP-43 in vivo. Mock-infected or PHEVinfected mice were stained with antiPHEV (red) and antiTDP-43 (green). Cells from the hippocampus are shown as examples. Scale bar $=100 \mu \mathrm{m}$ a

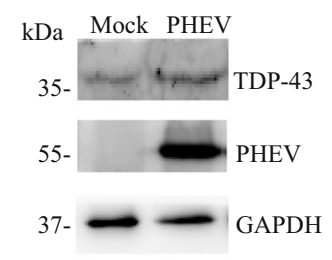

b

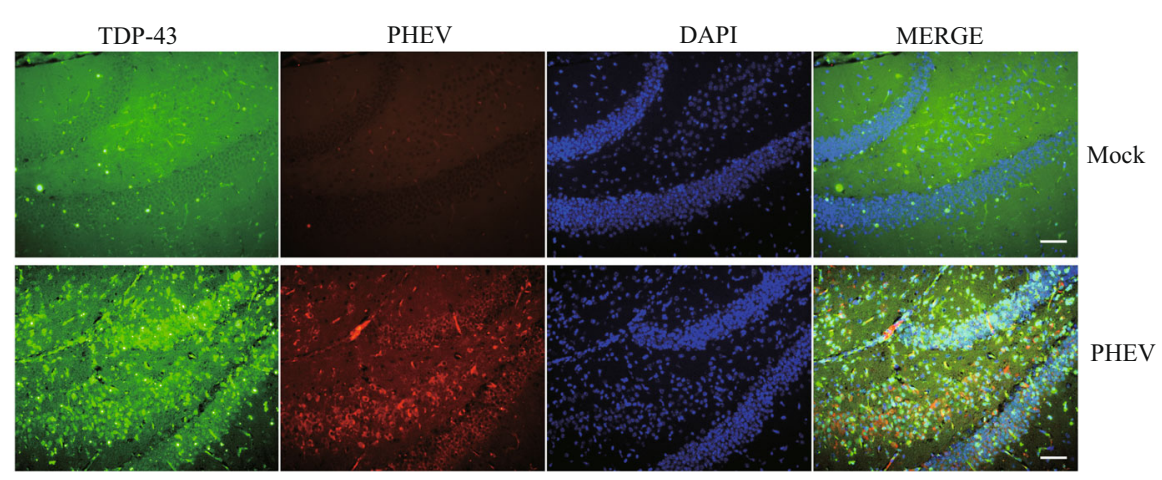


infection, and then enhancing lysosome capability to degrade pathogenic proteins (Additional file 5). These results suggest that PHEV may serve as an in vitro model for studying the pathogenic mechanisms and prevention of other degenerative diseases.

Neurodegeneration or neuronal loss by connecting with pathogenic proteins in a specific region of the brain represent a fundamental pathological feature across various neurodegenerative diseases in the pathological conditions of major neurodegenerative diseases such as Alzheimer's disease (AD), Parkinson's disease (PD), frontotemporal lobar degeneration (FTLD), and amyotrophic lateral sclerosis (ALS) [28]. PHEV infection was confirmed to be a risk factor for neurodegenerative diseases in our previous reports [9-11]. Acutely PHEV-infected mice showed typical neurological symptoms and frequently leaving behind grievous neurodegeneration [9-11]. Structural plasticity disorders occur in the axons, dendrites, and dendritic spines of PHEV-infected neurons, and PHEV-infected mice exhibit neuronal loss in the CNS [10]. In addition, acutely PHEV-infected mice were like human and mouse models of $G R N$ haploinsufficiency which recapitulate pathogenic lysosomal features of various neurodegenerative diseases (Additional files 1and 3). Furthermore, the transactive response DNA binding protein 43 (TDP-43)-associated proteinopathies related to PGRN expression have long been characterized as a main hallmark of ALS and FTLD with ubiquitin-positive inclusions (FTLD-U, also known as FTLDTDP). Indeed, we found that PHEV infection increased TDP43 protein expression in the whole brain of mice by western blotting analysis, especially in the hippocampus region by confocal fluorescence microscopy analysis (Fig. 3a, b) [29]. So, PHEV-infected mice represent a fundamental pathological feature of neurodegenerative diseases, and PHEV infection provided the opportunity to create animal models for these diseases. However, further research is needed to shed more light on the molecular mechanisms of the neurodegenerative lesions and changes in animal behavior observed in acutely, chronically and subclinically PHEV-infected mice.

\footnotetext{
Authors' Contributions Yungang Lan, $\mathrm{Zi} \mathrm{Li}$, and Wenqi He designed and supervised the experiments. Yungang Lan, Zhenzhen Wang, and Xinran Wang performed most of the tests. Gaili Wang, Kui Zhao, and Baofeng $\mathrm{Xu}$ cultured cells and analyzed immunofluorescence images. Jing Zhang and Shiyu Hu made brain tissue sections and stained. Yungang Lan drafted the manuscript. Wenqi He and Feng Gao revised the manuscript for valuable intellectual content. All authors read and approved the final manuscript.
}

Funding This study was supported by the National Key Research and Development Program of China (Grant 2016YFD0500102), the National Natural Science Foundation of China (Grants 31902262, 31872446, 31772704,31672519 , and 31602018), the State Key Laboratory of Genetically Engineered Veterinary Vaccines (no. AGVSKL- ZD201808), the China Postdoctoral Science Foundation (Grant 2020 M670858, 2018 M640285).
Data Availability All data generated or analyzed during this study are included in this published article and its supplementary information files.

\section{Compliance with Ethical Standards}

Ethical Approval and Consent All animal studies were conducted according to experimental practices and standards approved by the Animal Welfare and Research Ethics Committee of the College of Veterinary Medicine, Jilin University, China (permission number KT201904002), following the recommendations of the Council for International Organization of Medical Sciences on Animal Experimentation.

Consent for Publication Not applicable

Competing Interests The authors declare that they have no conflict of interest.

\section{References}

1. Gitler AD, Dhillon P, Shorter J (2017) Neurodegenerative disease: models, mechanisms, and a new hope. Dis Model Mech 10:499502

2. Li XQ, Sarmento L, Fu ZF (2005) Degeneration of neuronal processes after infection with pathogenic, but not attenuated, rabies viruses. J Virol 79:10063-10068

3. Volpi VG, Pagani I, Ghezzi S, Iannacone M, D'Antonio M, Vicenzi $\mathrm{E}$ (2018) Zika virus replication in dorsal root ganglia explants from interferon receptor1 knockout mice causes myelin degeneration. Sci Rep 8:10166

4. Cribbs DH, Azizeh BY, Cotman CW, LaFerla FM (2000) Fibril formation and neurotoxicity by a herpes simplex virus glycoprotein B fragment with homology to the Alzheimer's A beta peptide. Biochemistry 39:5988-5994

5. Jang H, Boltz D, Sturm-Ramirez K, Shepherd KR, Jiao Y, Webster R, Smeyne RJ (2009) Highly pathogenic H5N1 influenza virus can enter the central nervous system and induce neuroinflammation and neurodegeneration. Proc Natl Acad Sci U S A 106:14063-14068

6. Mora-Diaz JC, Pineyro PE, Houston E, Zimmerman J, GimenezLirola LG (2019) Porcine hemagglutinating encephalomyelitis virus: a review. Front Vet Sci 6:53

7. Hirano N, Nomura R, Tawara T, Tohyama K (2004) Neurotropism of swine haemagglutinating encephalomyelitis virus (coronavirus) in mice depending upon host age and route of infection. J Comp Pathol 130:58-65

8. Hirano N, Haga S, Sada Y, Tohyama K (2001) Susceptibility of rats of different ages to inoculation with swine haemagglutinating encephalomyelitis virus (a coronavirus) by various routes. J Comp Pathol 125:8-14

9. Lan Y, Zhao K, Zhao J, Lv X, Wang G, Lu H, Tang B, Li Z et al (2014) Gene-expression patterns in the cerebral cortex of mice infected with porcine haemagglutinating encephalomyelitis virus detected using microarray. J Gen Virol 95:2192-2203

10. Li Z, Zhao K, Lv X, Lan Y, Hu S, Shi J, Guan J, Yang Y et al (2018) Ulk1 governs nerve growth factor/TrkA signaling by mediating Rab5 GTPase activation in porcine hemagglutinating encephalomyelitis virus-induced neurodegenerative disorders. J Virol 92: 16

11. Li Z, Lan Y, Zhao K, Lv X, Ding N, Lu H, Zhang J, Yue H et al (2017) miR-142-5p disrupts neuronal morphogenesis underlying porcine hemagglutinating encephalomyelitis virus infection by targeting Ulk1. Front Cell Infect Microbiol 7:155 
12. Chen K, Zhao K, Song D, He W, Gao W, Zhao C, Wang C, Gao F (2012) Development and evaluation of an immunochromatographic strip for rapid detection of porcine hemagglutinating encephalomyelitis virus. Virol J 9:172

13. Holler CJ, Taylor G, McEachin ZT, Deng Q, Watkins WJ, Hudson K, Easley CA, Hu WT et al (2016) Trehalose upregulates progranulin expression in human and mouse models of GRN haploinsufficiency: a novel therapeutic lead to treat frontotemporal dementia. Mol Neurodegener 11:46

14. Zhou X, Sun L, Brady OA, Murphy KA, Hu F (2017) Elevated TMEM106B levels exaggerate lipofuscin accumulation and lysosomal dysfunction in aged mice with progranulin deficiency. Acta Neuropathol Commun 5:9

15. Zhou X, Sun L, Bastos de Oliveira F, Qi X, Brown WJ, Smolka MB, Sun Y, Hu F (2015) Prosaposin facilitates sortilin-independent lysosomal trafficking of progranulin. J Cell Biol 210:991-1002

16. Armstrong RA, Lantos PL, Cairns NJ (2008) What determines the molecular composition of abnormal protein aggregates in neurodegenerative disease? Neuropathology 28:351-365

17. Koh JY, Kim HN, Hwang JJ, Kim YH, Park SE (2019) Lysosomal dysfunction in proteinopathic neurodegenerative disorders: possible therapeutic roles of cAMP and zinc. Mol Brain 12:18

18. Xu B, Gao Y, Zhan S, Ge W (2017) Quantitative proteomic profiling for clarification of the crucial roles of lysosomes in microbial infections. Mol Immunol 87:122-131

19. Ding N, Zhao K, Lan Y, Li Z, Lv X, Su J, Lu H, Gao F et al (2017) Induction of atypical autophagy by porcine hemagglutinating encephalomyelitis virus contributes to viral replication. Front Cell Infect Microbiol 7:56

20. Bateman A, Bennett HP (2009) The granulin gene family: from cancer to dementia. Bioessays 31:1245-1254

21. Cenik B, Sephton CF, Kutluk Cenik B, Herz J, Yu G (2012) Progranulin: a proteolytically processed protein at the crossroads of inflammation and neurodegeneration. J Biol Chem 287:32298 32306

22. Kao AW, McKay A, Singh PP, Brunet A, Huang EJ (2017) Progranulin, lysosomal regulation and neurodegenerative disease. Nat Rev Neurosci 18:325-333

23. Almeida MR, Macario MC, Ramos L, Baldeiras I, Ribeiro MH, Santana I (2016) Portuguese family with the cooccurrence of frontotemporal lobar degeneration and neuronal ceroid lipofuscinosis phenotypes due to progranulin gene mutation. Neurobiol Aging 41:200 e201-200 e205

24. Snowden JS, Pickering-Brown SM, Mackenzie IR, Richardson AM, Varma A, Neary D, Mann DM (2006) Progranulin gene mutations associated with frontotemporal dementia and progressive nonfluent aphasia. Brain 129:3091-3102

25. Zhou X, Paushter DH, Feng T, Sun L, Reinheckel T, Hu F (2017) Lysosomal processing of progranulin. Mol Neurodegener 12:62

26. Mukherjee AB, Appu AP, Sadhukhan T, Casey S, Mondal A, Zhang Z, Bagh MB (2019) Emerging new roles of the lysosome and neuronal ceroid lipofuscinoses. Mol Neurodegener 14:4

27. Paushter DH, Du H, Feng T, Hu F (2018) The lysosomal function of progranulin, a guardian against neurodegeneration. Acta Neuropathol 136:1-17

28. Dawson TM, Golde TE, Lagier-Tourenne C (2018) Animal models of neurodegenerative diseases. Nat Neurosci 21(10):1370-1379

29. Ward ME, Chen R, Huang HY, Ludwig C, Telpoukhovskaia M, Taubes A, Boudin H, Minami SS et al (2017) Individuals with progranulin haploinsufficiency exhibit features of neuronal ceroid lipofuscinosis. Sci Transl Med 9:385

Publisher's Note Springer Nature remains neutral with regard to jurisdictional claims in published maps and institutional affiliations. 